\title{
Synthesis and supramolecular analysis of novel purine alkaloid cocrystals with trimesic and hemimellitic acids
}

\author{
M. Gołdyn, D. Larowska, E. Bartoszak-Adamska, W. Nowak \\ Adam Mickiewicz University, Uniwersytetu Poznańskiego 8, 61-614 Poznań \\ mateusz.goldyn@amu.edu.pl
}

Cocrystallization is becoming a more and more popular method to obtain new forms of drugs in the pharmaceutical industry. In this way, their physicochemical properties, like solubility, bioavailability, permeability through biological membranes, stability can be modified without affecting their pharmacological properties [1]. Cocrystals are homogeneous solids consisting of components in a neutral or ionic form, which are solids under ambient conditions, in a specific stoichiometric ratio. Such combinations of APIs (active pharmaceutical ingredients) with appropriately selected coformers are defined as pharmaceutical cocrystals [2].

The main goal of the study was to use purine alkaloids, such as theobromine, theophylline, and caffeine for cocrystallization with trimesic (TMSA) and hemimellitic acid (HMLA) [3]. Theobromine forms cocrystals TBR·TMSA and TBR·HMLA. Caffeine forms

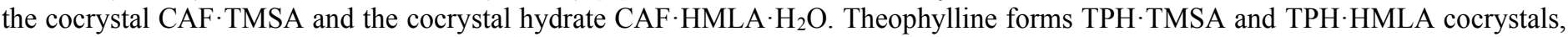
the cocrystal hydrate TPH$\cdot T M S A \cdot 2 \mathrm{H}_{2} \mathrm{O}$ and the salt hydrate $(\mathrm{TPH})^{+} \cdot(\mathrm{HMLA})^{-} \cdot 2 \mathrm{H}_{2} \mathrm{O}$. The reactions were carried out in solution and by neat or liquid-assisted grinding in a ball mill. Powder analysis showed that 7 out of 8 solids were obtained by mechanochemical synthesis. All obtained multicomponent complexes were structurally characterized by the single-crystal X-ray diffraction method. The use of compounds with slight structural differences allowed the investigation of the complexity of specific non-covalent interactions formation. Selected coformers can form strong hydrogen bonds with the carboxyl groups participation, therefore 3 types of supramolecular synthons have been distinguished: alkaloid-alkaloid, alkaloid-acid and acid-acid synthons.

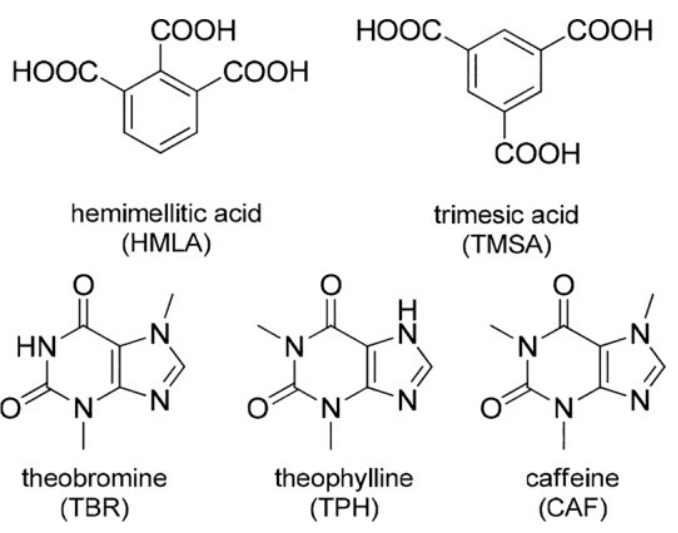

Figure 1. Purine alkaloids and tricarboxylic acids used for cocrystallization.

$\mathrm{X}$-ray structural analysis showed the dominant role of alkaloid-acid and acid-acid interactions. These studies also show that it is sometimes possible to predict what non-covalent interactions will be responsible for the arrangement of molecules in the crystal lattice of the synthesized complex. However, the study of the self-assembly processes of molecules in systems with many functional groups is well-founded as the complexity of supramolecular synthons shows that the crystal structure design is often laborious.

Additionally, UV-Vis measurements determined the effect of the cocrystallization of purine alkaloids on their solubility in water.

[1] Kumar S., Nanda A. (2017), Pharmaceutical Cocrystals: An Overview, Indian J. Pharm. Sci., 79, 858-871.

[2] Duggirala N. K., Perry M. L., Almarsson Ö., Zaworotko M. J. (2016), Pharmaceutical cocrystals: along the path to improve medicines, Chem. Commun., 52, 640-655.

[3] Gołdyn M., Larowska D., Bartoszak-Adamska E. (2021), Novel Purine Alkaloid Cocrystals with Trimesic and Hemimellitic Acids as Coformers: Synthetic Approach and Supramolecular Analysis, Cryst. Growth Des., 21, 396-413.

Keywords: cocrystals; purine alkaloids; grinding cocrystallization; supramolecular synthons; X-ray structural analysis

The work was supported by grant no. POWR.03.02.00-00-I026/16 co-financed by the European Union through the European Social Fund under the Operational Program Knowledge Education Development.

Acta Cryst. (2021), A77, C1202 